\title{
Pratica interdisciplinar de ensino-aprendizagem simulando o efeito chicote em uma Cadeia de Suprimentos
}

\author{
Interdisciplinary teaching-learning practice simulating the bullwhip effect in a \\ supply chain
}

\author{
Andrey Sartori ${ }^{1 *}$, Rubens de Oliveira ${ }^{1}$, Bárbara Perez ${ }^{1}$, Anderson Vieira ${ }^{1}$, Carla Arcanjo ${ }^{1}$, \\ Daniel Maciel ${ }^{1}$, Rosicley de Siqueira ${ }^{1}$, Fabricio de Moraes $^{2}$
}

\begin{abstract}
RESUMO
A realização de simulações no ensino superior tem ganhado espaço como uma alternativa na assimilação dos conceitos teóricos ensinados em salas de aula. Essa pratica proporciona ao aluno a sensação de um ambiente real e vivenciado dentro das empresas. Sendo assim, ampliar a sala de aula para além das suas paredes é uma forma de trazer o problema atual para dentro da classe, mostrando o problema prático e partindo para solução teórica. A simulação tem como intenção estimular o estudante a desenvolver uma visão sistêmica e analítica dos problemas existentes ao longo de uma Cadeia de Suprimentos, gerando uma percepção sobre a importância da teoria acadêmica para solucionar problemas organizacionais reais. Esse artigo tem como objetivo demonstrar uma pratica interdisciplinar de Ensino-Aprendizagem a respeito do efeito chicote na Cadeia de Suprimentos, envolvendo a disciplina de Gestão da Cadeia de Suprimentos em um curso de Mestrado Profissional da UFRGS. Os resultados coletados foram analisados e discutidos pelos alunos e o professor, demonstrando que o efeito chicote na Cadeia de Suprimentos é real, porém suas consequências podem ser mitigadas quando bem utilizadas às ferramentas tecnológicas e as informações disponíveis.
\end{abstract}

Palavras-chave: Simulação; Efeito Chicote; Cadeia de Suprimentos.

\begin{abstract}
The realization of simulations in higher education has gained space as an alternative in the assimilation of theoretical concepts taught in classrooms. This practice gives the student the feeling of a real and experienced environment within companies. Therefore, extending the classroom beyond its walls is a way of bringing the current problem into the class, showing the practical problem and starting with a theoretical solution. The simulation is intended to encourage the student to develop a systemic and analytical view of the problems that exist throughout a Supply Chain, generating a perception of the importance of academic theory to solve real organizational problems. This article aims to demonstrate an interdisciplinary Teaching-Learning practice regarding the bullwhip effect in the Supply Chain, involving the discipline of Supply Chain Management in a Professional Masters course at UFRGS. The collected results were analyzed and discussed by the students and the teacher, demonstrating that the bullwhip effect in the Supply Chain is real, but its consequences can be mitigated when well used to the technological tools and the available information.
\end{abstract}

Keywords: SWOT Matrix; Internal Environment; External Environment; Strategic Plan.

\footnotetext{
${ }^{1}$ Instuição de afiliação: FATEC SENAI MT

*E-mail: andrey.sartori@ senaimt.edu.br

${ }^{2}$ Instuição de afiliação diferente: IFMT
} 


\section{INTRODUÇÃO}

O processo de ensino é uma ação intencional, uma vez que, exige que outras ações sejam desenvolvidas com o apoio de teorias e tendências pedagógicas que direcionam a prática docente, logo se torna necessário planejamento, objetivo, seleção de conteúdos, métodos aplicáveis, recursos e por fỉm criar estratégias de avaliação dessas ações, gerando uma reflexão sobre a relação teoria e prática. A educação como provedora de conhecimentos teóricos para auxiliar na Gestão da Cadeia de Suprimentos tem assumido um papel importante dentro das Instituições de Ensino Superior - IES.

Conforme Tubino e Schafranski (2000), a simulação através de jogos permite que ideias e conceitos, passíveis de serem aplicados na prática, sejam testados de uma maneira mais simples, possibilitando a avaliação dos impactos desses e a escolha das estratégias mais adequadas para cada situação.

O objetivo desse artigo é demonstrar uma prática interdisciplinar de EnsinoAprendizagem a respeito do ensino no Curso Superior de Logística envolvendo a disciplina de Gestão da Cadeia de Suprimentos simulando uma pratica do efeito chicote através do jogo Easter Game. Buscando assim estimular o estudante a criar uma visão sistêmica e analítica dos problemas empresariais, gerando uma percepção da importância da teoria acadêmica para solucionar problemas organizacionais reais.

\section{REFERÊNCIAL TEÓRICO \\ RELAÇÕES DO ENSINO TEÓRICO COM A PRÁTICA}

A visão tradicional e secular do ensino, ou seja, do professor ministrando a teoria expositiva aos alunos em sala de aula e cobrando o aprendizado com provas e trabalhos, conforme estudos demonstra ser pouco produtiva ao desenvolvimento acadêmico e a formação profissional do estudante. Mesmo com a possibilidade de realizar perguntas e tirar dúvidas individuais, a aula teórica pouco agrega de valor a formação desse futuro profissional.

Conforme Kallás (2003), esse método não é ao todo equivocado, porém necessita de um complemento para criar uma visão e um pensamento sistêmico organizacional mais consistente e seguro, gerando segurança nas tomadas de decisões 
dentro das empresas quando necessário com base nos conhecimentos adquiridos, habilidades que são cada vez mais exigidas pelo mercado de trabalho.

\section{ESTRATÉGIAS DE ENSINO}

Para Belhot (1997); Silva \& Cecílio (2007), as estratégias de ensino devem ir além da utilização de exemplos e figuras. As aulas teóricas e carregadas de conteúdo desestimulam o aluno que percebe pouca relação entre a teoria e a prática. Sendo assim, o professor passa a ter um papel mais atuante na transmissão do conhecimento e um corresponsável no processo de aprendizagem.

Outra vantagem das práticas educacionais está em equacionar as diferenças de velocidade de aprendizagem, os alunos com maior dificuldade de entendimento de conteúdo, passam a entender melhor utilizando estratégias práticas de aprendizagem. É uma forma de lidar com as diferentes formas de apresentar e organizar as informações (WANKAT \& OREOVICZ,1993).

\section{EASTER GAME}

O "Easter Game" é um clássico baseado no jogo da cerveja ou "Beer Game”, um jogo de empresas criado por John Sterman, do MIT Sloan School of Management na década de 60. Seu objetivo é simular o comportamento de uma Cadeia de Suprimentos com demanda flutuante. Os participantes do jogo podem enviar pedidos de mercadorias para fornecedores, e têm acesso apenas à sua informação local, desconhecendo a informação dos demais elos da Cadeia de Suprimentos, causando o chamado efeito chicote. O "Easter Game” serve ao propósito de 'simular' alguns dos fenômenos típicos que ocorrem nas Cadeias de Suprimentos. O entendimento desses fenômenos é condição fundamental para a discussão das questões relacionadas à configuração e ao planejamento e programação das Cadeias de Suprimentos.

\section{EFEITO CHICOTE}


O efeito chicote pode ser compreendido como a amplificação da demanda ao longo da Cadeia de Suprimentos, uma forma de demonstrar a percepção por trás do comportamento dinâmico das Cadeias de Suprimentos, são os erros decorrentes da propagação da demanda junto à Cadeia de Suprimentos, originando assim uma amplificação dessa demanda. Este fenômeno já havia sido percebido (Mitehel, 1923) pelos economistas, no início do século XX, cujo comportamento da amplificação é, em geral, não linear ao longo da cadeia (GEARY et al., 2006). Pode-se exemplificar seu comportamento de forma gráfica:

Figura 1. Ilustração gráfica do efeito chicote numa cadeia de abastecimentos fictícia

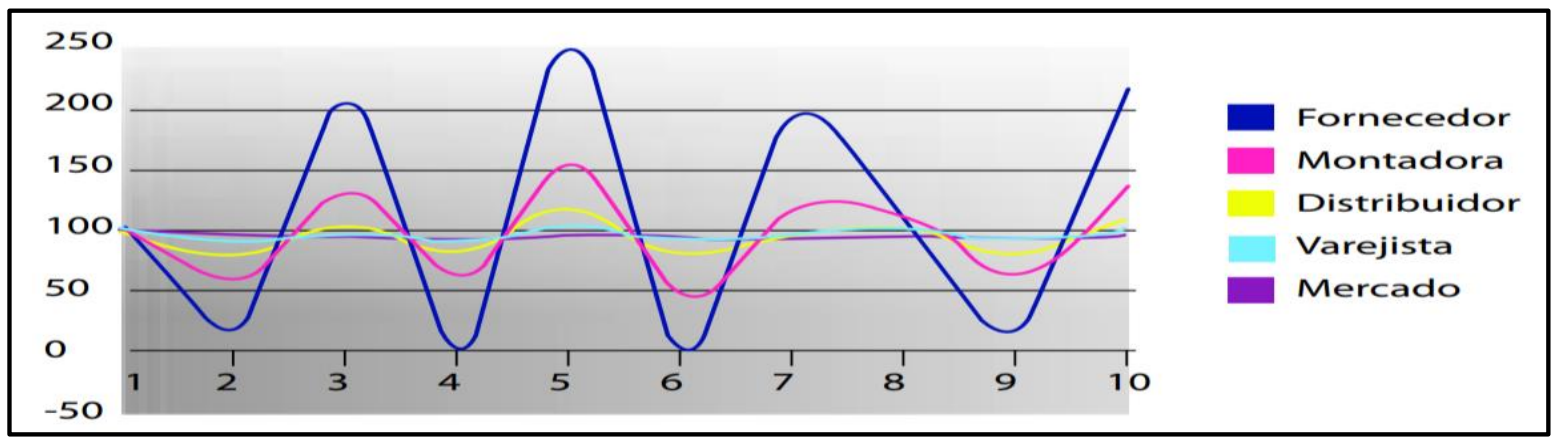

Fonte: adaptado de Slack (1999)

Segundo Lee et al (1997), as principais causas do Efeito Chicote são: (i) atualização da demanda futura; (ii) acúmulo de pedidos; (iii) flutuação do preço; e (iv) racionalização e "jogo da demanda". Estes mesmos autores elencam algumas formas para evitar o efeito chicote e categorizam as diversas formas em Sincronização da Informação, Alinhamento de Canais e Eficiência Operacional.

\section{METODOLOGIA}

Com a evolução da metodologia do ensino superior sob uma forma geral, os jogos e exercícios de simulação saíram da exclusividade dos cursos com temática gerencial e administrativa, e foram implantados em outras áreas de ensino, tal como psicologia, marketing, economia, educação física, entre outras. 
Gramigna (1993) apresenta a seguinte sugestão para classificar os diferentes tipos de jogos: Jogos de Comportamento são aqueles cujo tema central permite que se trabalhem temas voltados às habilidades comportamentais; Jogos de Processo são os jogos onde a ênfase maior é dada às habilidades técnicas; Jogos de Mercado são os que reúnem as mesmas características dos jogos deprocesso, mas são direcionados para atividades que reproduzem situações demercado.

A partir da classificação mencionada, a simulação que será apresentada neste artigo se enquadra melhor em jogos de mercado, pois melhor atende os objetivos descritos, o método adotado possui caráter descritivo, apresentando uma pesquisa bibliográfica e pôr fim à abordagem será quali-quantitativa.

Antes de iniciar o jogo, os alunos foram divididos em grupos representando os agentes de uma Cadeia de Suprimentos, sendo esses: Fornecedor > Indústria > Distribuidor $>$ Atacado $>$ Varejo $>$ Cliente final. Cada grupo assume seu papel no jogo, sendo o professor o demandante principal (cliente final). Conforme o demandante inicia sua solicitação de produtos, a Cadeia de Suprimentos responde atendendo ou não a solicitação, os valores são anotados em uma planilha para posterior discussão dos resultados.

Foram simuladas 8 rodadas em um prazo de 22 semanas e os dados históricos utilizados foram aleatórios, conforme acontece na realidade em uma Cadeia de Suprimentos sem gestão. Os grupos não podiam se comunicar uns como os outros durante todo o exercício de simulação. Valores como quantidade em estoque, estoque final e inicial, quantidade pedida pelo agente sucessor da cadeia e quantidade solicitada ao outro agente fornecedor eram anotadas em uma planilha para controle das equipes.

A partir da experiência do jogo "Easter Game", pede-se que cada equipe analise os resultados obtidos e reflita acerca dos conceitos envolvidos e de possíveis redesenhos de processos que pudessem levar a um desempenho superior. Como resultado, pede-se que sejam elaborados um relatório e uma breve apresentação por cada grupo, que devem abordar:

- Planejamento

- Quais são as principais decisões tomadas pelos jogadores ao longo do jogo?

- Como foi estruturada a lógica de decisão da sua equipe? 
- Problemas

- Quais são os principais problemas observados?

- Como esses estão relacionados?

- Resultados do jogo

- Padrões de demanda e pedidos;

- Custos de estocagem e backlog;

- Demanda verificada versus planejamento com atenuação exponencial;

- Jogo x realidade

- Supondo uma situação real, que outros aspectos deveriam ser considerados?

- Como eles se diferenciam de acordo com os tipos de mercado e sistemas de produção?

- Quais são as alterações e/ou melhorias que a equipe sugere?

\section{RESULTADOS E DISCUSSÕES}

\section{PLANEJAMENTO}

A equipe buscou usar de estratégias para se preparar e tentar tomar as melhores decisões possíveis, já que não existiam algumas informações importantes como:
a) a data exata em que aconteceria a pascoa;
b) a quantidade de estoques do nosso atacadista, distribuidor e da fábrica;
c) não havia histórico de vendas anteriores;
d) a demanda do mercado inicial era desconhecida;
e) as decisões foram tomadas em curto prazo.

Com a impossibilidade dos grupos se comunicarem com os outros estágios da cadeia, analisou-se a informação disponível que era a solicitação de pedidos do cliente e o quanto se possuía em estoque inicial, considerando o lead time de entrega dos pedidos que era de 1 semana e a variação nas quantidades solicitadas pelo cliente. Outro fator 
importante e relevante para as decisões foram os custos de estoque e "Backlog", esse mais alto do que o de se manter estoques.

Conforme o jogo foi criando forma, a equipe percebeu que nem sempre o atacadista possuía produtos para nos atender de forma integral, essa nova informação vinda agora do processo que acontecia para trás da cadeia, nos fez tomar medidas de precaução e algumas vezes foram solicitados mais produtos do que a média ponderada normal apontava, até que o atacadista pudesse se estabilizar e atender os pedidos. $\mathrm{O}$ uso da média ponderada ajudou a equipe a estabelecer as quantidades que seriam solicitadas ao atacadista e estabelecer um estoque mínimo de produtos e com isso minimizar o risco de não atendimento do cliente já que o custo de não atende-lo seria o dobro de se manter os produtos em estoque.

\section{PROBLEMAS}

A falta de comunicação entre os agentes da cadeia e de informações relevantes gerou uma maior insegurança durante as decisões do grupo. Isso minimizou que ações fossem tomadas com maior sucesso. A comunicação entre os elos da cadeia é fundamental para se estabelecer ajustes de estoques e atender previsões de demanda, pois o custo de se manter estoques desnecessários pode tornar o produto não competitivo e todas as causas imprevistas tem o poder de alimentar a efeito chicote ao longa da cadeia. A visão de compartilhamento de informações ou pelo menos o estreitamento das comunicações entre os elos de cada estágio pode ajudar a reduzir a oscilação do efeito chicote.

Outro problema relacionado está na geração de estoques e o custo relacionado. Existem empresas que mantem os estoques altos com medo de não conseguir atender os clientes buscando evitar a insatisfação, e minimizar o risco de perder alguns dos seus consumidores para concorrência. Na simulação foi possível verificar isso no feedback do jogo, onde verificou-se que todos os envolvidos finalizaram a simulação com estoques desnecessários, resultado da falta de sincronia entres a cadeia.

Porém, o simples fato de compartilhar informação das demandas não é suficiente, já que cada elo da cadeia pode adotar diferentes métodos de previsão, baseando-se no nos seus pontos fortes ou fracos. Cada um possui suas particularidades 
de suprimentos, transportes, capacidade de armazenamento, limite de produção e distribuição, e porque não, até da situação financeira favorável ou não, em que a empresa se encontra no momento. Leva-se em consideração ainda a necessidade de todas terem a mesma capacidade empresarial e tecnológica de ação e gestão.

A tecnologia é fundamental para que o compartilhamento de dados seja eficiente e rápido. Já a gestão precisa ser alinhada e de forma objetiva para que todos os componentes da cadeia tenham um mesmo modelo. Isso ajuda a diminuir o lead time de

pedidos e transmite mais segurança para todos os envolvidos. É possível verificar que uma das principais causas de geração de estoques na Cadeia de Suprimentos está na falta de relação entre os agentes, gerando instabilidade e perda de confiança na rede.

\section{RESULTADOS DO JOGO}

Os padrões de demanda e pedidos não são homogêneos e variam muito de acordo com as solicitações enviadas ao longo de toda cadeia, originando-se no consumidor. O efeito chicote (EC) são observações nas quais a variabilidade da demanda é aumentada tendo como foco a solicitação do cliente e percorre toda cadeia até chegar à fábrica, porem sua ampliação acontece por vários fatores em cada estágio da cadeia, esse processo causa instabilidade na cadeia e o aumento de custos de estocagem e fornecimento ao cliente final.

Essa distorção na cadeia, mostra em muitos casos a falta de informações programadas, um lapso temporal da propagação da informação entre o consumo no ponto de venda e os agentes da cadeia. Conforme se verificou os padrões de demanda e pedidos eram inconstantes como acontece em situações não simuladas. O nível de variabilidade da demanda aumentava à medida que avançava os níveis da cadeia, onde nem sempre os pedidos de compra eram atendidos de forma integral, isso gerava insegurança na equipe 3 e um aumento nos pedidos e a geração de estoques para não deixar de atender as demandas dos clientes.

Os custos de não atender o cliente eram maiores que os custos de se manter o estoque. Por isso, a necessidade de se manter um equilíbrio nos pedidos feitos para trás da cadeia. O Gráfico 1, mostra a demanda da média ponderada realizada pela equipe para poder estipular a quantidade pedida ao distribuidor. 
Gráfico 1. Comportamento da demanda em relação à média ponderada

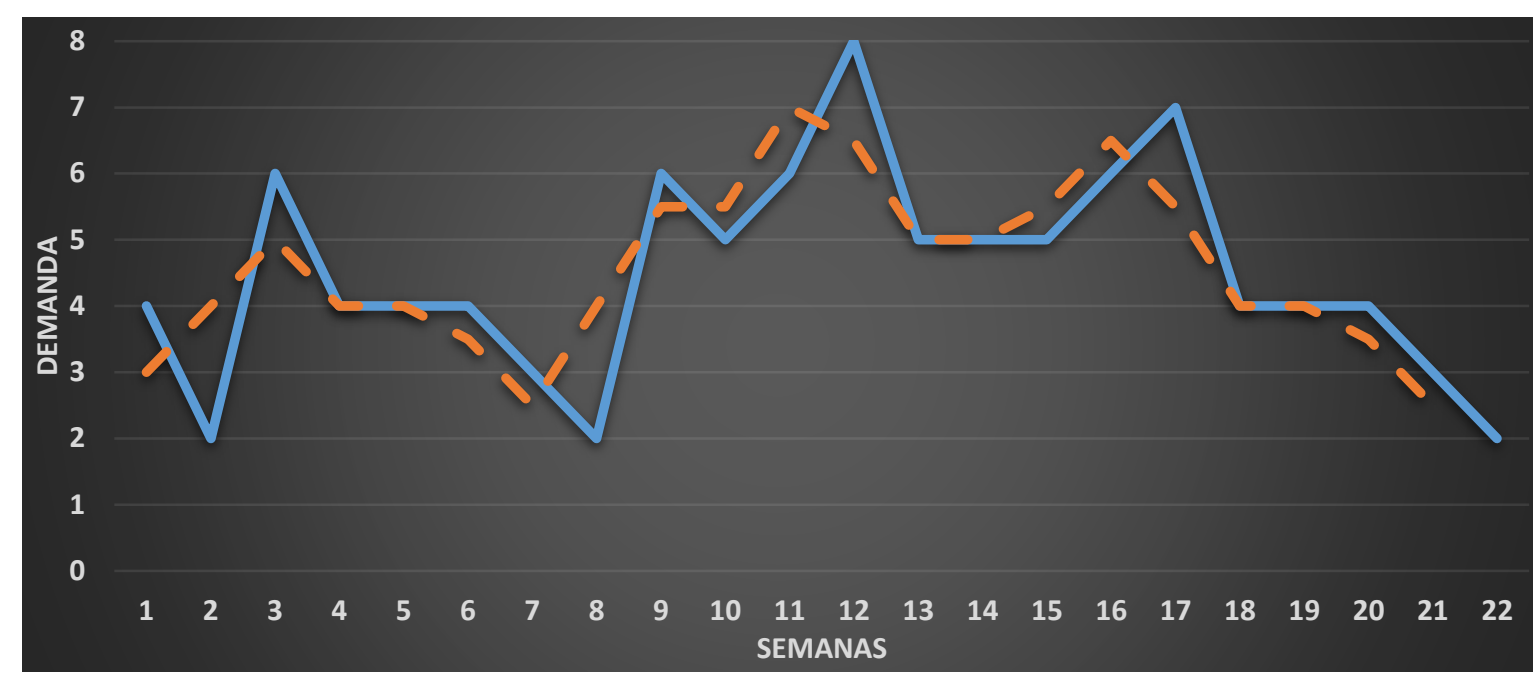

Fonte: autores, 2016

Armstrong (2002) descreve que um dos motivos para erros na previsão ocorre quando as estimativas consideram fontes de dados com grau de incerteza considerável. O autor afirma, contudo, que se existem dados estatísticos suficientes, métodos quantitativos são preferíveis, pois em geral são mais precisos que os métodos qualitativos. Os gráficos a seguir mostram como a previsão de demanda (linha pontilhada) do jogo "Easter Game" se comporta com relação a dados históricos (linha contínua), apoiado na média ponderada.

\section{JOGO VERSUS REALIDADE}

Supondo uma situação real, é possível analisar outros aspectos. No Gráfico 2 é possível verificar uma outra simulação, feita a partir dos dados do jogo, onde foram dados três valores para $(\mathrm{N})$, ou seja, foram calculadas as médias ponderadas com base em 3 semanas, 6 semanas e 9 semanas, para se chegar a uma melhor analise de qual média seria mais viável e flutuaria menos.

O Gráfico 2 demonstra que a média ponderada $(\mathrm{N}=9)$ reflete um valor médio melhor da demanda, já que o erro médio é zero, porém seu desvio padrão é maior. Quando o valor fica em $(\mathrm{N}=3)$ se tem um valor bem próximo de zero no erro médio ($0,2)$, e apresenta um desvio padrão menor em relação à média ponderada $(\mathrm{N}=9)$. Sendo assim, o gráfico mais suave e linear é a média $(\mathrm{N}=6)$, pois é o que apresenta o menor desvio padrão, sendo o mais ideal para previsão de demanda dos meses subsequentes. 
Gráfico 2. Analise das previsões de demanda com valores médios para (N)

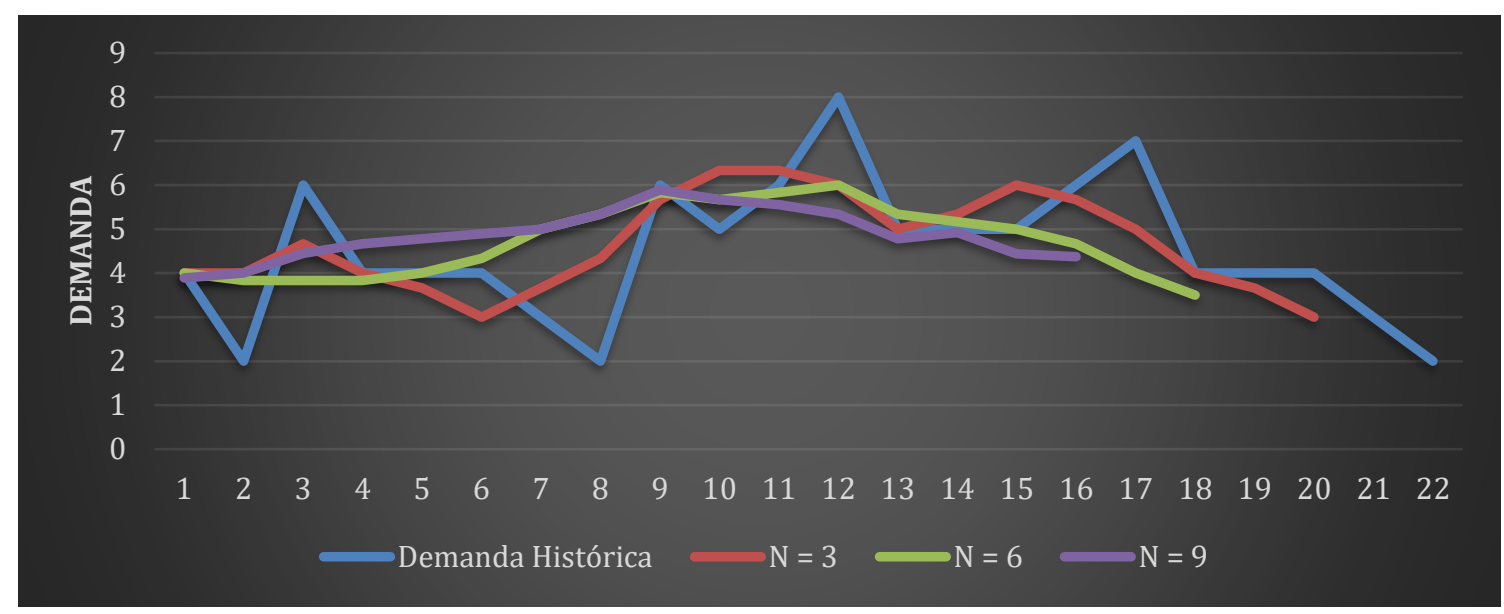

\begin{tabular}{|c|c|c|c|}
\hline Média Ponderada (N) & $(\mathrm{N}=3)$ & $(\mathrm{N}=6)$ & $(\mathrm{N}=9)$ \\
\hline Previsão para mês 23 & 3,0 & 4,0 & 4,9 \\
\hline Previsão para mês 24 & 2,7 & 3,5 & 4,4 \\
\hline Previsão para mês 25 & 2,6 & 3,4 & 4,4 \\
\hline Erro médio & $-0,2$ & $-1,3$ & 0,0 \\
\hline Desvio Padrão & 1,56 & 1,3 & 1,9 \\
\hline
\end{tabular}

Fonte: Autores, 2016

\section{SUAVIZAÇÃO EXPONENCIAL SIMPLES}

Esse modelo é usado quando a demanda não apresenta tendência ou sazonalidade para decisões de curto prazo, o seu valor está, restrito ao intervalo [0;1], utilizando a formula: $\mathrm{Ft}=\mathrm{Ft}-1+\alpha($ At-1 $-\mathrm{Ft}-1)$.

$$
\begin{aligned}
\text { Onde: } & \\
F_{t} & =\text { Previsão para o periodo } t \\
A_{t-1} & =\text { valor real para o periodo } t-1 \\
F_{t-1} & =\text { Previsăo para o periodo } t-1 \\
\alpha & =\text { Constante de alisamento }
\end{aligned}
$$

Com analise da demanda em relação ao planejamento com suavização exponencial, utilizam-se valores de alfa para atenuação das previsões de demandas anteriores. No Gráfico 3, que mostra outra simulação de como se poderia realizar a 
análise da demanda histórica prevista para cada modelo utilizando a atenuação exponencial. A linha do menor desvio padrão é (Alfa $=4)$, apresentando assim, uma linha mais suave ao longo do gráfico, o que demonstra uma previsão mais linear.

Gráfico 3. Atenuação Exponencial

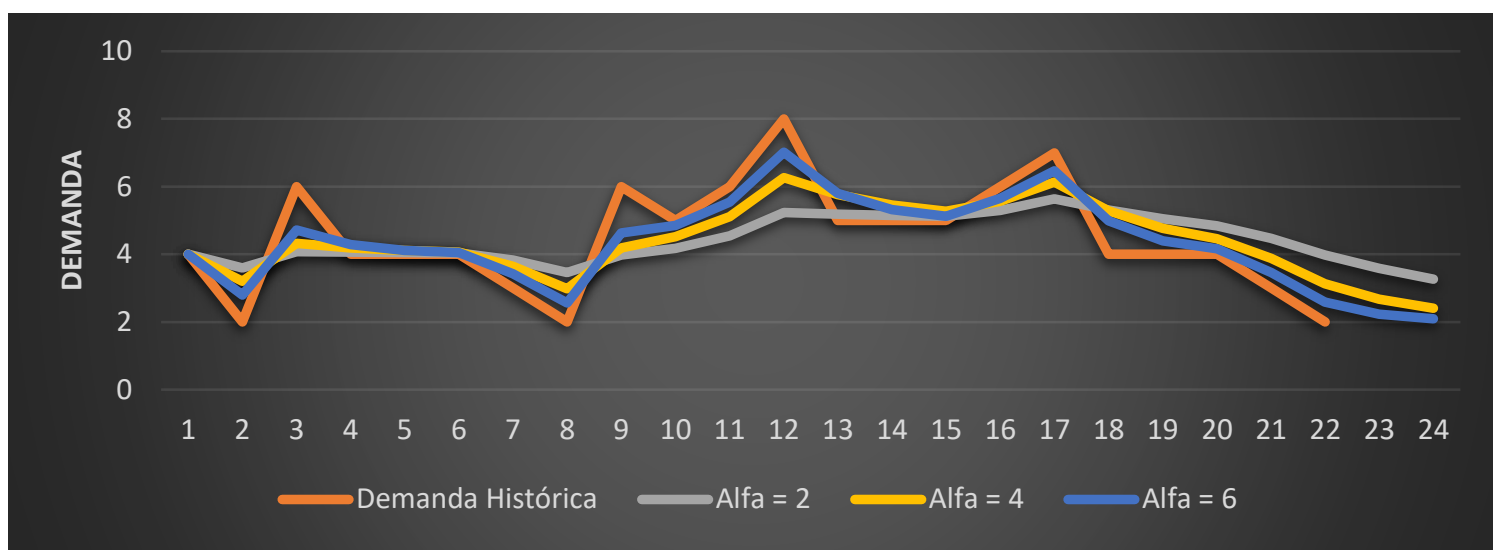

\begin{tabular}{|c|c|c|c|}
\hline Atenuação Exponencial & $\alpha=2$ & $\alpha=4$ & $\alpha=6$ \\
\hline Previsão para mês 23 & 4,0 & 3,1 & 2,6 \\
\hline Previsão para mês 24 & 3,6 & 2,7 & 2,2 \\
\hline Previsão para mês 25 & 3,3 & 2,4 & 2,1 \\
\hline Erro médio & 0,1 & 0,0 & 0,0 \\
\hline Desvio Padrão & 1,65 & 1,62 & 1,64 \\
\hline
\end{tabular}

Fonte: autores, 2016

Existem muitos aspectos a serem considerados em uma situação real, fatores que podem ou não surtir resultados, mas não podem ser deixados de lado. $\mathrm{O}$ acesso a demanda final verdadeira é um bom princípio para se considerar, isso evitaria previsões múltiplas e ciclos de pedidos muito longos, melhorando o processamento da previsão de demanda. Tentar buscar um planejamento único em toda cadeia. Esse talvez seja um ponto difícil de realizar, tendo em vista as questões particulares de cada elo da cadeia, conforme sugerem os autores Lamming et al. (2000) e Pires (2004).

Os conceitos se diferenciam de acordo com os agentes envolvidos da Cadeia de Suprimentos, independente do tamanho da cadeia, dos tipos de mercado e/ou sistemas de produção. Sendo assim, são sugeridas as seguintes ações de melhorias: Aplicação de tecnologias da informação como ERP, para integrar a cadeia, assim melhorando o fluxo 
de informações; Melhor flexibilidade, diminuição no prazo de entrega, desta forma atendendo mais rápido nosso cliente final; Melhor estratégia de distribuição; Entrega com distribuidor e varejista, escolheria um distribuidor com menor prazo possível; Controle através de indicadores de desempenho: percebe-se neste setor de produtos avarias nos ovos de páscoa entregue aos consumidores, onde de maneira legal a indústria e toda a cadeia de distribuição são responsáveis em realizar troca do produto.

Muito embora com estas alterações/melhorias tivessem estoques mais ajustados, poder-se-ia doar os ovos de páscoa não vendidos para instituições de caridade, no intuito de maximizar a marca do lojista perante mecanismos de publicidade; Ter mais de um fornecedor para atender o prazo; Realizar acordos de níveis de serviço entre os elos da cadeia; Compra programada para obter escala e assim trabalhar a redução de preço e a garantia de atendimento. Melhorando o fluxo de estoques em toda cadeia.

\section{CONCLUSÃO}

Com as respostas dadas as questões apresentadas pelo professor e os conhecimentos aprendidos para resolução dos problemas foi possível verificar que o chamado efeito chicote da Cadeia de Suprimentos acontece com mais frequência do que se imagina. É importante que ações sejam tomadas para tentar buscar minimizar esse efeito, tais como, ter uma visão sistêmica da Cadeia de Suprimentos, percebendo as relações de interdependência entre os elos que a compõe, buscar o planejamento integrado e as vantagens da colaboração entre empresas que compõe uma mesma Cadeia de Suprimentos, adquirir uma nova percepção dos custos envolvidos na gestão de estoques e do nível de serviço ao cliente como orientadores da estratégia da empresa, buscar um equilíbrio na coordenação entre demanda e recursos da organização disponíveis para o atendimento do cliente, melhorar a comunicação das informações relevantes aos processos da cadeia, no que se refere à demanda e produção, melhorar a gestão de estoques dos elos envolvidos da cadeia, evitar flutuações de preços para não gerar efeitos que desbalancem a lei da oferta e da demanda. Esses fatores não são únicos, é preciso considerar outros problemas que dependeram do tipo Cadeia de Suprimentos existente e dos fatores relevantes envolvidos em cada processo.

Cada cadeia deve buscar os seus objetivos e tentar equilibrar as reações causadas, buscando a minimização do efeito chicote. A utilização de técnicas de ensino 
baseadas em simulações apresentam bons resultados quanto ao desenvolvimento intelectual dos participantes, proporcionando melhor entendimento da teoria utilizando a simulação como aplicação prática do conteúdo, facilitando a assimilação de conceitos que serão aplicados ao longo de sua vida profissional. Mas prever as reações dos participantes à proposta de aula ainda é uma dificuldade ao se preparar e realizar as simulações, onde alguns alunos podem apresentar aversão ao método adotado devido à falta de interesse ou motivação. Devido a esse fator, fica evidente a importância de promover outras simulações ao longo da formação, a fim de obter maior participação, proporcionando experiência com a metodologia e visando melhores resultados.

\section{REFERÊNCIAS}

BALLOU, Ronald H. Gerenciamento da cadeia de suprimentos/logística empresarial /Ronald H. Ballou, tradução Raul Rubenich. - 5.ed. - Porto Alegre: Bookman, 2006. Reimpressão 2009, 616 p.; 28 cm. ISBN 978-85-363-0591-2.

CASSEL, Ricardo A. Logística Empresarial. (2016) Apostila da Disciplina de Logística Empresarial e gestão da Cadeia de Suprimentos, Porto Alegre: UFRGS/IBG.

CHEN et al. Quantifying the bullwhip effect in a simple supply chain: the impacto $f$ forecasting, lead times, and information. Management science, v.46, n.3, p. 436-433, 2000 .

COELHO, Leandro Callegari; FOLLMANN, Neimar. O impacto do compartilhamento de informações na redução do efeito chicote na cadeia de suprimentos. Gestão e Produção, São Carlos, v.16, n. 4, p.571-583, out.-dez, 2009.

COELHO, Leandro Callegari; FOLLMANN, Neimar; RODRIGUES, Carlos Manuel. O Efeito Chicote e o seu Impacto na Gestão da cadeia de Suprimentos. IV Simpósio de Excelência em Gestão e Tecnologia, 2007.

FIORIOLLI, J. C. Modelagem matemática do Efeito Chicote em cadeias de abastecimento. 2007. Tese (Doutorado em Engenharia) - Universidade Federal do Rio Grande do Sul. FORRESTER, J. Industrial Dynamics. Harvard Business Review, n. 36, Julho - Agosto 1958.

GRAMIGNA, M.R.M. Jogos de Empresa. São Paulo, Makron Books, 1993.

Grupo de Estudos Logísticos - UFRGS/IBG. Rondonópolis, Mato Grosso: Mestrandos do Curso de Engenharia de Produção e Inovação, Universidade Federal do Rio Grande do Sul - UFRGS, 2016.

KALLÁS, D. A Utilização de Jogos de Empresas no Ensino da Administração. In: VI SEMEAD - Seminários em Administração, VI, 2003. 
LEE, H. et al. The bullwhip effect in supply chains. Sloan Management Review, n.38, p. 93-102, 1997.

SLACK, Nigel; CHAMBERS, Stuart; JOHNSTON, Robert. Administração da

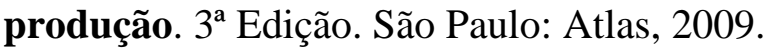

TUBINO, D \& SCHAFRANSKI, L.E. Simulação Empresarial em Gestão da Produção. Manual de Simulação. Universidade Federal de Santa Catarina, 2000.

Recebido em: 10/10/2021

Aprovado em: 20/11/2021

Publicado em: 25/11/2021 